\section{Association between obesity profile and non-alcoholic fatty liver by race/ ethnicity}

\author{
Martin Galindo, Katrina M Schrode and Magda Shaheen*
}

Office of Research, Charles R. Drew University, Los Angeles, USA

\section{Abstract}

NAFLD is characterized by accumulation of fat in the liver that can lead to health complications Previous studies have found the obesity phenotype and its components to be risk factors for the development of NAFLD. This study aims to examine the relationship between the obesity phenotype and NAFLD among each racial-ethnic group. We analyzed data from the NHANES III survey (1988-1994). The obesity phenotype was defined based on BMI and metabolic syndrome. NAFLD was defined by abdominal ultrasounds among non-alcoholics with no infection or taking drugs affecting the liver. A higher prevalence of NAFLD was found among the metabolically unhealthy obese group (43.1\%) and the metabolically unhealthy overweight $(29.4 \%)$ than the metabolically unhealthy normal weight $(11.8 \%)$. Mexicans-Americans had higher odds of NAFLD relative to whites (adjusted odds ratio $(A O R)=1.3,95 \%$ confidence interval $(\mathrm{Cl})=1.01-1.9$, $p=0.04)$. The metabolically healthy obese phenotype was associated with NAFLD $(p>0.05)$ in the overall sample and in Whites. The metabolically healthy overweight was associated with NAFLD only among Mexican-American $(p<0.05)$. Metabolically unhealthy overweight or obese had higher odds of NAFLD relative to the metabolically healthy normal weight and this relation is consistent in all the racial/ethnic groups $(p<0.05)$. Metabolically healthy overweight and obese individuals had a high chance of NAFLD and it varied by race/ethnicity. Healthcare providers should pay more attention to care for those who are part of the metabolically healthy overweight or obese group especially among the Mexican-American population.

\section{More Information}

*Address for Correspondence:

Magda Shaheen, Charles R. Drew University, 1731 E 120th St, Los Angeles, CA 90059, USA, Tel: (323) 357-3453;

Email: magdashaheen@cdrewu.edu; mshaheen@ucla.edu

Submitted: January 08, 2021

Approved: January 18, 2021

Published: January 19, 2021

How to cite this article: Galindo M, Schrode KM Shaheen M. Association between obesity profile and non-alcoholic fatty liver by race/ethnicity. Ann Clin Endocrinol Metabol. 2021; 5: 001-010.

DOI: 10.29328/journal.acem.1001017

ORCiD: orcid.org/0000-0001-9077-5798 Copyright: @ 2021 Galindo M, et al. This is an open access article distributed under the Creative Commons Attribution License, which permits unrestricted use, distribution, and reproduction in any medium, provided the original work is properly cited.

Check for updates

OPEN ACCESS

\section{Introduction}

Fatty liver disease is typically characterized by resistance to insulin along with fat accumulation in the liver [1,2]. Nonalcoholic fatty liver disease (NAFLD) refers to fatty liver disease that is not related to excessive alcohol consumption, drug consumption, or liver injury caused by herbal products $[1,2]$. NAFLD is the most common cause of liver disease in the world with a prevalence of about $30 \%$ in developed countries [1]. Patients with NAFLD often develop comorbidities that can lead to a bigger burden being placed on the healthcare system. If not treated, NAFLD can develop into NASH which often leads to advance liver disease, hepatocellular carcinoma (HCC), and cirrhosis; consequently, NASH is now considered the second biggest risk factor in determining who needs a liver transplant [3].

NAFLD primarily affects those who are obese, but also affects patients who are lean [4]. Associations have been found between hepatic steatosis and metabolic syndrome and its components, including central obesity based on waist circumference, insulin resistance, dyslipidemia, hypertension, and hypertriglyceridemia [2,5-7]. The prevalence of metabolic syndrome in NAFLD patients in one study was $41.3 \%$ [8]. Patients with both metabolic syndrome and NAFLD have also been found to have a higher risk of developing NASH and fibrosis of the liver [1].

The obesity phenotype refers to classification based on metabolic health and obesity. Body mass index (BMI) is typically used to classify obesity [9]. While obesity and metabolic syndrome are highly correlated, some individuals have only one of the risk factors and not the other. Thus, obesity phenotype can ranging from metabolically unhealthy normal weight to metabolically healthy obese [10-12]. A metabolically obese phenotype, for example, would be characterized by elevated body mass index (BMI) and a healthy metabolic profile. There is limited research investigating how obesity phenotype affects risk for NAFLD. Chang, et al. [13] found that in a cohort of metabolically healthy obese individuals, obesity was progressively associated with incidence of NAFLD, while 
Shaharyar, et al. [14] found that incidence of hepatic steatosis was higher in metabolically healthy obese subjects compared to metabolically healthy normal weight subjects. On the other hand, in studies of non-obese subjects, increases in the number of diagnosed metabolic components corresponded with increased prevalence of NAFLD $[15,16]$. These studies highlight the importance of looking at both components of the obesity phenotype to assess risk for NAFLD.

Previous studies have indicated that NAFLD varies by race/ ethnicity. Several studies have found higher NAFLD prevalence in Hispanics and lower prevalence in Blacks when compared to non-Hispanic whites [17-19]. A growing literature is showing that genetic risk factors, which are unequally distributed among the different racial/ethnic groups, are associated with the development of NAFLD [20,21]. Studies investigating racial disparities in obesity phenotype are lacking. However, a recent study by the Center for Disease Control and Prevention (CDC) has shown the highest prevalence of obesity to occur in non-Hispanic Blacks, followed by Hispanics [22]. Previous studies have also observed differences in the prevalence of metabolic syndrome in Hispanics compared to other groups, but results have been more mixed and strongly impacted by sex [23-25].

This study's aim was to examine the relationship between the obesity phenotype and the prevalence of NAFLD in the United States population among each racial/ethnic group. We used data from Third National Health and Nutrition Examination Survey (NHANES III). While these data were collected several years ago, they remain important because they constitute a nationally representative sample of the U.S. population and use ultrasound for the diagnosis of liver steatosis. It is imperative that medical providers are aware of how obesity phenotype affects the risk for liver disease, and particularly how this risk factor differs between racial/ ethnic groups so that they can screen and monitor patients appropriately.

We hypothesized that metabolically healthy overweight/ obese individuals will be more likely to have NAFLD relative to the metabolically healthy normal weight individuals. In addition, this relation is more likely to occur in MexicanAmericans relative to Whites.

\section{Materials and methods}

\section{Data source}

We analyzed data from the Third National Health and Nutrition Examination Survey (NHANES III) 1988-1994 which is a cross-sectional survey using multistage stratified sample of a representative sample of the non-institutionalized population of the United States to examine the health and nutrition of children and adults.

The survey protocol was approved by the NCHS Research Ethics Review Board and was in accordance with the
Declaration of Helsinki [26]. Informed consent was obtained from all participants prior to participation. The details of the NHANES III procedures can be found in the article that included the program and collections procedure as well as the plan and operation of the study [27].

The Third NHANES study took place over 6 years and involved 33,994 participants aged 2 months and older. Initial interviews were completed at the participants' residences, while physical and laboratory examinations were conducted at mobile examination centers [27]. NHANES III oversampled Mexican Americans, non-Hispanic blacks, persons 60 years and older, and children ages two months to five years. We analyzed data from NHANES III for adults aged 20 years and older with ultrasound data.

\section{Main independent variables}

The independent variables for this study are the obesity phenotype and race/ethnicity. Obesity phenotype was categorized by BMI and metabolic syndrome, creating six levels: metabolically healthy normal weight; metabolically healthy overweight; metabolically healthy obese; metabolically unhealthy normal weight; metabolically unhealthy overweight; metabolically unhealthy obese. Obesity classification was defined as follows: Normal weight BMI $<25 \mathrm{~kg} / \mathrm{m}^{2}$, overweight BMI $\geq 25$ and $<30 \mathrm{~kg} / \mathrm{m}^{2}$ and obese $\mathrm{BMI} \geq 30 \mathrm{~kg} / \mathrm{m}^{2}$. Metabolically healthy was defined as having 1 or fewer components of metabolic syndrome, while unhealthy was having more than 1 component. The components consideredwere: 1)Systolicblood pressure (SBP $\geq 140 \mathrm{~mm}-\mathrm{Hg}$ ) and/or diastolic blood pressure (DBP $\geq 90 \mathrm{~mm}-\mathrm{Hg}$ ) or current drug treatment for hypertension. 2) Waist circumference $>88 \mathrm{~cm}$ for women, $>102 \mathrm{~cm}$ for men. 3) Fasting plasma glucose $\geq 100 \mathrm{mg} / \mathrm{dl}(5.6 \mathrm{mmol} / \mathrm{l})$ or current drug treatment for diabetes. 4) $\mathrm{HDL}<50 \mathrm{mg} / \mathrm{dl} \quad(1.29 \mathrm{mmol} / \mathrm{l})$ for women, $<40 \mathrm{mg} / \mathrm{dl}(1.03 \mathrm{mmol} / \mathrm{l})$ for men or current drug treatment for high cholesterol. 5) Fasting triglycerides $\geq$ $150 \mathrm{mg} / \mathrm{dl}(1.7 \mathrm{mmol} / \mathrm{l})$ or current drug treatment for high triglycerides. Race and ethnicity were grouped into the following categories: non-Hispanic white, non-Hispanic Black, Mexican American, or other.

\section{Dependent variable}

The dependent variable for this study was NAFLD. Participants 20 years and older in the NHANES III underwent abdominal ultrasonography. In NHANES III, the ultrasound recordings were analyzed and subjects were rated as having no, mild, moderate, or severe hepatic steatosis. For the purposes of our study, cases with moderate to severe steatosis were classified as having hepatic steatosis. Participants were considered to have NAFLD if they had hepatic steatosis and did not have any exclusion criteria. Exclusion criteria included elevated transferrin level $>50 \%$, chronic hepatitis B, chronic hepatitis C, excessive alcohol use, or prescription medications that might cause hepatic steatosis [28-30]. Chronic hepatitis B 
was defined as positive results for both the hepatitis B surface antigen and hepatitis B core antibody tests. Chronic hepatitis C was defined as positive results for both the hepatitis $\mathrm{C}$ antibody and RNA tests. Excessive alcohol use was defined as an average of more than 2 drinks/day for men or 1 drink/ day for women. Average alcohol use was determined by multiplying the responses to the two questions: "Number of days drank alcohol in past 12 months" and "average drinks per day on drinking day" and dividing by 365 to get a daily average.

\section{Confounding variables}

We included potential confounding factors for hepatic steatosis and NAFLD based on literature review. The following variables were included in the analyses: demographics (age, sex, education, urbanization, and poverty), physical activity status, smoking status, laboratory values (cholesterol, HbA1c, HOMA insulin resistance (IR), C-reactive protein (CRP), alanine aminotransferase (ALT), aspartate aminotransferase (AST), and C-peptide), hypertension and healthy eating index (HEI).

Age was measured in years in NHANES III. Sex was recorded as either female or male. Education level was categorized as less than the $12^{\text {th }}$ grade, completed $12^{\text {th }}$ grade, and completed past the $12^{\text {th }}$ grade. Language spoken at home was classified as English, Spanish, both English and Spanish, or other. Alcohol consumption by participants was recorded as never, former, and current.

Urbanization was classified as urban if the subject lived in a metro area containing at least 1 million people, and rural otherwise. Federal poverty ratio was defined as the family income divided by the federal poverty threshold and classified as $<1,1-2$, and $>2$. Physical activity was assessed by asking subjects how often they engaged in a variety of recreational or other activities requiring physical exertion. Subjects who reported not doing any of the activities were classified as inactive. National guidelines at the time of data collection recommended doing moderate activity at least 5 times/week or vigorous activity at least 3 times/week. We further classified active subjects based on these guidelines into active \& meets guidelines and active \& does not meet guidelines. Smoking was classified as never, former, or current. C-peptide levels were classified as low $(<0.26 \mathrm{nmol} / \mathrm{L})$, normal $(0.26-1.03 \mathrm{nmol} / \mathrm{L})$, and high $(>1.03 \mathrm{nmol} / \mathrm{L})$. Total cholesterol was categorized as normal $(\leq 200 \mathrm{mg} / \mathrm{dL})$, elevated (200-239 $\mathrm{mg} / \mathrm{dL}$ ), and high ( $\geq 240 \mathrm{mg} / \mathrm{dL})$. Triglyceride levels were categorized as normal $(<150 \mathrm{mg} / \mathrm{dL})$, borderline $(150-190$ $\mathrm{mg} / \mathrm{dL})$, or high $(\geq 200 \mathrm{mg} / \mathrm{dL})$. Glucose was categorized as normal $(<100 \mathrm{mg} / \mathrm{dL})$, prediabetes $(100-125 \mathrm{mg} / \mathrm{dL})$, or diabetes $(>125 \mathrm{mg} / \mathrm{dL}$ ). ALT was categorized as normal $(\leq 56 \mathrm{U} / \mathrm{L})$ or elevated $>56 \mathrm{U} / \mathrm{L})$. AST was categorized as normal $(\leq 40 \mathrm{U} / \mathrm{L})$ or elevated $>40 \mathrm{U} / \mathrm{L}$ ). CRP was categorized as normal $(0.1-<0.3 \mathrm{mg} / \mathrm{dL}$ ), mild (mild inflammation) $(0.3-1 \mathrm{mg} / \mathrm{dL})$, and high (significant inflammation) $(>1 \mathrm{mg} / \mathrm{dL})$.

\section{Statistical analysis}

The main independent variables in this study were obesity phenotype and race/ethnicity. The dependent variable was NAFLD. Population characteristics are presented using descriptive statistics. Categorical variables are presented as unweighted numbers and weighted percent. We examined the differences in population characteristics and NAFLD outcome by the independent variables using Chi-square tests. We used multiple logistic regression to determine the relationship between the obesity phenotype and NAFLD status for each racial/ethnic group and adjusted for the other independent variables. We present the data as adjusted odds ratio and $95 \%$ confidence interval and p-value of $<0.05$ is considered statistically significant. We analyzed the data using SAS (Release V.9.1.3, 2002; SAS, Inc) and the survey module of STATA (Release V.10, 1984e2007 Statistics/Data Analysis; StataCorp). The NCHS provided sample weights that we used to correct for differential selection probabilities and to adjust for non-coverage and non-response. All estimates were weighted as supplied by NHANES and the design has been taken into consideration.

\section{Results}

\section{Population characteristics}

We analyzed data from 13,060 people who participated in NHANES III. Table 1 shows the population characteristics. The largest groups was metabolically healthy normal weight (36.3\%). The metabolically healthy obese represented 3.9\%; $19.1 \%$ were metabolically unhealthy obese, $7.9 \%$ were metabolically unhealthy normal weight, $17.5 \%$ were metabolically unhealthy overweight, and $15.3 \%$ were metabolically healthy overweight.

Most of the participants were White (75.5\%) followed by a smaller percentage of Blacks (11\%) and Mexican-Americans (5.5\%). Mexican-Americans had the highest prevalence of metabolically healthy overweight $(18.9 \%)$ compared to Whites (14.9\%) and Blacks (17.7\%). Mexican-Americans had a higher prevalence of metabolically unhealthy overweight (19\%) when compared to Whites (18\%) and Blacks (14.7\%). Blacks had the highest prevalence of metabolically healthy obese (7.5\%) when compared to Whites (3.4\%) and MexicanAmericans (3.8\%). Blacks also had a higher prevalence of the metabolically unhealthy obese $(23.3 \%)$ when compared to Whites (18.5\%) and Mexicans (23.2\%).

\section{Prevalence of NAFLD}

The prevalence of NAFLD in the population was $18.5 \%$. The highest prevalence of NAFLD was found in the metabolic unhealthy obese group (43\%), followed by $29.4 \%$ among the metabolic unhealthy overweight group, and $18.5 \%$ among the metabolic healthy $(p<0.05)$. NAFLD prevalence was also found to be higher in Mexican-Americans (25.5\%) compared to whites (17.8\%), and lowest in Blacks (14.7\%). 
Table 1: Overall population characteristics and by non-alcoholic fatty liver status.

\begin{tabular}{|c|c|c|c|c|c|c|c|}
\hline \multirow[t]{2}{*}{ Variables } & \multirow{2}{*}{$\begin{array}{c}\text { Total } \\
\text { Number }\end{array}$} & \multirow[b]{2}{*}{ Weighted percent } & \multicolumn{2}{|c|}{ NAFLD $(n=2790,8.5 \%)$} & \multicolumn{2}{|c|}{ Normal/Mild Steatosis $(n=10745,81.7 \%)$} & \multirow[t]{2}{*}{$p$ - value } \\
\hline & & & Number & Weighted Percent & Number & Weighted Percent & \\
\hline Obesity Phenotype & & & & & & & 0.0001 \\
\hline Metabolically healthy normal weight & 3976 & 36.3 & 307 & 6.2 & 3606 & 93.8 & \\
\hline Metabolically healthy overweight & 2003 & 15.3 & 236 & 8 & 1727 & 92 & \\
\hline Metabolically healthy obese & 585 & 3.9 & 103 & 18.5 & 472 & 81.5 & \\
\hline Metabolically unhealthy normal weight & 1062 & 7.9 & 158 & 11.8 & 877 & 88.2 & \\
\hline Metabolically unhealthy overweight & 2532 & 17.5 & 695 & 29.4 & 1750 & 70.6 & \\
\hline Metabolically unhealthy obese & 2998 & 19.1 & 1199 & 43.1 & 1668 & 56.9 & \\
\hline Age (years) & & & & & & & 0.0001 \\
\hline 20-34 & 5024 & 37.23 & 656 & 11.6 & 4284 & 88.4 & \\
\hline $35-49$ & 3958 & 32.33 & 843 & 17.9 & 2989 & 82.1 & \\
\hline $50+$ & 4928 & 30.44 & 1291 & 26 & 3472 & 74 & \\
\hline Race/Ethnicity & & & & & & & 0.0001 \\
\hline White & 5068 & 75.53 & 979 & 17.8 & 3961 & 82.2 & \\
\hline Black & 4094 & 10.98 & 622 & 14.7 & 3388 & 85.3 & \\
\hline Mexican-American & 4164 & 5.48 & 1085 & 25.5 & 2930 & 74.5 & \\
\hline Other & 584 & 8.01 & 104 & 18.6 & 466 & 81.4 & \\
\hline Sex & & & & & & & 0.0009 \\
\hline Male & 6508 & 48.48 & 1365 & 19.6 & 4889 & 80.4 & \\
\hline Female & 7402 & 51.52 & 1425 & 16.4 & 5856 & 83.6 & \\
\hline Urban/rural & & & & & & & 0.009 \\
\hline Urban & 7051 & 48.76 & 1303 & 16.1 & 5552 & 83.9 & \\
\hline Rural & 6859 & 51.24 & 1487 & 19.7 & 5193 & 80.2 & \\
\hline Language spoken at home & & & & & & & 0.0005 \\
\hline English & 11000 & 89.39 & 1969 & 17.8 & 8358 & 82.2 & \\
\hline Spanish & 2795 & 6.262 & 727 & 24.2 & 1975 & 75.9 & \\
\hline Both & 323 & 4.012 & 48 & 16.9 & 135 & 83.1 & \\
\hline Other & 194 & 0.3342 & 46 & 12.1 & 275 & 87.9 & \\
\hline Federal poverty level & & & & & & & 0.3346 \\
\hline$<1$ & 3434 & 20.39 & 666 & 19.9 & 2256 & 80.1 & \\
\hline 1 to 2 & 3022 & 12.61 & 696 & 18.9 & 2630 & 81.1 & \\
\hline$>2$ & 6238 & 66.99 & 1157 & 17.3 & 4946 & 82.7 & \\
\hline Smoking status & & & & & & & 0.0001 \\
\hline Current & 3951 & 29.79 & 612 & 13.8 & 3183 & 86.2 & \\
\hline Former & 3195 & 24.94 & 817 & 24.2 & 2263 & 75.8 & \\
\hline Non-smoker & 6763 & 45.26 & 1361 & 17.3 & 5298 & 82.7 & \\
\hline Physical activity & & & & & & & 0.0001 \\
\hline Inactive & 3107 & 15.62 & 748 & 22.1 & 2260 & 77.9 & \\
\hline Does not meet guideline & 5488 & 40.6 & 1162 & 19.6 & 4178 & 80.4 & \\
\hline Meets guidelines & 5315 & 43.77 & 880 & 14.9 & 4307 & 85.1 & \\
\hline Education grade completed & & & & & & & 0.0001 \\
\hline less than high school & 4406 & 34.45 & 1318 & 22.8 & 3818 & 77.2 & \\
\hline high school & 5329 & 23.28 & 814 & 18.8 & 3482 & 81.2 & \\
\hline more than high school & 4086 & 42.27 & 644 & 14.7 & 3373 & 85.3 & \\
\hline Total cholesterol & & & & & & & 0.0001 \\
\hline $\operatorname{good}(<200 \mathrm{mg} / \mathrm{dL})$ & 6544 & 50.15 & 1063 & 13.8 & 5321 & 86.2 & \\
\hline elevated (200-239 mg/dL) & 4172 & 31.13 & 960 & 20.5 & 3099 & 79.6 & \\
\hline high (> = $240 \mathrm{mg} / \mathrm{dL})$ & 2566 & 18.72 & 655 & 25.2 & 1820 & 74.8 & \\
\hline Triglyceride & & & & & & & 0.0001 \\
\hline normal (< 150 mg/dL) & 8998 & 68.89 & 1223 & 10.7 & 7573 & 89.3 & \\
\hline borderline (150-199 mg/dL) & 2397 & 17.59 & 511 & 26 & 1288 & 74 & \\
\hline high (> = 200 mg/dL) & 1854 & 13.52 & 942 & 41.1 & 1349 & 58.6 & \\
\hline C-reactive protein (CRP) & & & & & & & 0.0001 \\
\hline normal $(0.1-<0.3 \mathrm{mg} / \mathrm{dL})$ & 8766 & 72.45 & 1448 & 15 & 7112 & 85 & \\
\hline mild inflammation (0.3-1 mg/dL) & 3302 & 21 & 925 & 26.3 & 2261 & 73.7 & \\
\hline significant inflammation (> $1 \mathrm{mg} / \mathrm{dL}$ ) & 1138 & 6.54 & 291 & 26.4 & 805 & 73.6 & \\
\hline Serum glucose & & & & & & & 0.0001 \\
\hline normal (<100 mg/dL) & 9625 & 77.22 & 1512 & 14.1 & 7905 & 85.9 & \\
\hline prediabetes (100-125 mg/dL) & 972 & 4.83 & 698 & 26.8 & 1778 & 73.2 & \\
\hline diabetes (> $125 \mathrm{mg} / \mathrm{dL})$ & 2579 & 17.96 & 439 & 51.4 & 437 & 48.6 & \\
\hline Aspartate amino transferase (AST) & & & & & & & 0.0001 \\
\hline normal $(<=40 \mathrm{U} / \mathrm{L})$ & 12000 & 96.3 & 2441 & 17.5 & 9784 & 82.5 & \\
\hline elevated (> $40 \mathrm{U} / \mathrm{L})$ & 655 & 3.7 & 210 & 36.6 & 338 & 63.4 & \\
\hline Alanine amino transferase (ALT) & & & & & & & 0.0001 \\
\hline
\end{tabular}




\begin{tabular}{|c|c|c|c|c|c|c|c|}
\hline normal $(<=56 \mathrm{U} / \mathrm{L})$ & 13000 & 97.8 & 2511 & 17.5 & 9941 & 82.5 & \\
\hline elevated (> $56 \mathrm{U} / \mathrm{L})$ & 385 & 2.203 & 140 & 46 & 181 & 54 & \\
\hline \multicolumn{8}{|l|}{ Healthy eating index score (HEI) } \\
\hline poor diet $(<50)$ & 1323 & 10.86 & 462 & 17.8 & 1842 & 82.2 & \\
\hline needs improvement $(50-80)$ & 9814 & 73.37 & 1984 & 17.8 & 7551 & 82.2 & \\
\hline good diet $(80-100)$ & 2367 & 15.77 & 276 & 19.5 & 1026 & 80.5 & \\
\hline Hypertension & & & & & & & 0.0001 \\
\hline Yes & 3720 & 21.9 & 1081 & 30.5 & 2480 & 69.5 & \\
\hline No & 10000 & 78.1 & 1686 & 14.6 & 8147 & 85.4 & \\
\hline C-peptide & & & & & & & 0.0001 \\
\hline low $(<0.26 \mathrm{nmol} / \mathrm{L})$ & 1760 & 14.99 & 104 & 4.6 & 1624 & 95.4 & \\
\hline normal $(0.26-1.03 \mathrm{nmol} / \mathrm{L})$ & 8578 & 66.36 & 1339 & 13.6 & 7068 & 86.4 & \\
\hline high (> 1.03 nmol/L) & 3010 & 18.65 & 1242 & 45.4 & 1604 & 54.6 & \\
\hline
\end{tabular}

\section{Multiple logistic regression analysis}

Table 2 shows the multiple logistic regression analysis of NAFLD and obesity phenotype after adjustment for confounding variables and table 3 shows the stratified analysis by race/ethnicity. The overall results of the multiple logistic regression analysis adjusting for the confounding variables indicated that Mexican-Americans had a greater odds of having NAFLD than whites $(\mathrm{AOR}=1.38,95 \% \mathrm{CI}=1.01-1.9, p=0.04)$, and while Blacks had a lower chance of NAFLD than whites, the difference was not statistically signficant. Compared to the metabolically healthy normal weight, the metabolically unhealthy obese adults had the highest odds of NAFLD (AOR $=3.85,95 \% \mathrm{CI}=2.79-5.31, p<.0001)$, followed by the metabolically healthy obese $(\mathrm{AOR}=2.68,95 \% \mathrm{CI}=1.51-4.8$, $p=.001)$ and the metabolically unhealthy overweight (AOR = $2.5,95 \% \mathrm{CI}=1.86-3.37, p=<.0001)$.

Whites showed a relationship between obesity phenotype and NAFLD that was consistent to the overall pattern, where compared with the metabolically healthy normal weight, the highest odds of NAFLD were in the unhealthy obese group $(\mathrm{AOR}=4.14,95 \% \mathrm{CI}=2.78-6.16, p=<.0001)$, followed by the healthy obese $(\mathrm{AOR}=3.31,95 \% \mathrm{CI}=1.48-7.41, p=.004)$ and the unhealthy overweight $(\mathrm{AOR}=2.75,95 \% \mathrm{CI}=1.92-3.93$, $p=<0.0001$ ).

For Blacks, the only group that had a significantly elevated risk of NAFLD compared to the metabolically healthy normal weight were the metabolically unhealthy obese $(\mathrm{AOR}=1.86$, $95 \% \mathrm{CI}=1.32-2.63, p=.001$ ). Most other groups actually had a reduced risk of NAFLD, but none of these differences were statisically significant.

In Mexican-Americans, compared to the healthy normal weight group, all other obesity phenotypes had increased odds of NAFLD, although these differences were not statistically significant for the metabolically unhealthy normal weight or the metabolically healthy obese. The largest odds was in the metabolically unhealthy obese $(\mathrm{AOR}=3.85,95 \% \mathrm{CI}=2.33$ $6.38, p=<.0001$ ), followed by the metabolically unhealthy overweight $(\mathrm{AOR}=2.06,95 \% \mathrm{CI}=1.37-3.09, p=0.001)$ and the metabolically healthy overweight $(\mathrm{AOR}=1.52,95 \% \mathrm{CI}=$ 1.14-2.03, $p=0.005$ ).
The confounding variables showed consistent effects across the racial/ethnic groups except for sex and age. Overall, females were less likely to have NAFLD than males $(\mathrm{AOR}=.82$, $95 \% \mathrm{CI}=.70-.97, p=.021$ ), and the risk for NAFLD increased in older age groups, with a significant difference in the $50+$ group compared to those $20-34(\mathrm{AOR}=1.40,95 \% \mathrm{CI}=1.08-1.82$, $p=0.012$ ). When stratified by racial/ethnic group, only Mexican-Americans showed significantly lower odds in females compared to males $(\mathrm{AOR}=.69,95 \% \mathrm{CI}=.53-.89$, $p=.006)$. Similarly, only in Mexican-Americans did those in the 35-49 group (AOR $=1.58,95 \% \mathrm{CI}=1.18-2.13, p=0.003$ ) and $50+$ group $(\mathrm{AOR}=1.42,95 \% \mathrm{CI}=1.02-1.97, p=0.038)$ have higher odds of NAFLD than those in the 20-34 group.

\section{Discussion}

The purpose of this study was to examine the relationship between the obesity phenotype and NAFLD in each racial/ ethnic group in the US population. In the overall population, we found an independent association between the obesity phenotype and NAFLD where metabolically healthy and unhealthy obese individuals had a higher chance of NAFLD relative to metabolically healthy normal weight individuals. This finding is consistent with previous work that has found that metabolically healthy and metabolically abnormal obese individuals are both at high risk for hepatic steatosis [31,32]. We also found that metabolically unhealthy overweight individuals had a higher chance of NAFLD compared to the metabolically healthy normal weight, showing the importance of normal weight maintenance and metabolic health. Together, these results indicate that neither component of the obesity phenotype alone is sufficient to determine risk for NAFLD, but that the entire obesity phenotype must be considered.

Mexican-Americans had the highest prevalence of NAFLD, while Blacks had the lowest. We saw consistently higher odds of NAFLD in the metabolically unhealthy obese group relative to the metabolically healthy normal weight in all racial/ethnic groups. Among the Black population, none of the other obesity phenotypes increased risk for NAFLD. Both whites and Hispanics had increased odds of NAFLD in the metabolically unhealthy overweight group compared to the metabolically healthy normal weight group. However, only whites had increased odds of NAFLD in the metabolically healthy obese 
Table 2: Adjusted odds ratio and 95\% confidence intervals for significant associations with NAFLD based on multiple logistic regression

\begin{tabular}{|c|c|c|c|c|}
\hline Outcome: non-alcoholic fatty liver & Adjusted Odds Ratio & $\begin{array}{c}\text { Lower } 95 \% \\
\text { Confidence level }\end{array}$ & $\begin{array}{c}\text { Upper } 95 \% \\
\text { confidence level }\end{array}$ & $p$ - value \\
\hline
\end{tabular}

Obesity phenotype

Metabolically healthy overweight versus metabolically healthy normal weight

Metabolically healthy obese versus metabolically healthy normal weight

Metabolically unhealthy normal weight versus metabolically healthy normal weight

Metabolically unhealthy overweight versus metabolically healthy normal weight

Metabolically unhealthy obese versus metabolically healthy normal weight

Female versus male

Race/ethnicity

Black versus white

Mexican-American versus white

Other versus white

Rural versus urban

Language spoken at home

Spanish versus English

Both English and Spanish versus English

Other versus English

Smoking status

Current versus never

Former versus never

Age group (years)

$35-49$ versus $20-34$ years

$50+$ versus $20-34$ years

Healthy eating index group needs improvement versus good

poor diet versus good

Cholesterol groups

elevated (200-239 mg) versus normal

high ( $>=240 \mathrm{mg} / \mathrm{dL}$ ) versus normal

Triglyceride groups

borderline (150-199) versus normal

high ( $>=200 \mathrm{mg} / \mathrm{dL}$ ) versus normal

Glucose groups

diabetes (> $125 \mathrm{mg} / \mathrm{dL}$ ) versus normal

prediabetes (100-125) versus normal

ALT groups

elevated (> $56 \mathrm{U} / \mathrm{L}$ ) versus normal

AST groups

elevated (> $40 \mathrm{U} / \mathrm{L}$ ) versus normal

Education level

high school versus more than high school

less than high school versus more than high school CRP groups

mild inflammation versus normal

significant inflammation versus normal

C-peptide groups

high (>1.03 $\mathrm{nmol} / \mathrm{L}$ ) versus normal

low $(<.26 \mathrm{nmol} / \mathrm{L})$ versus normal

Hypertension

yes versus no

Poverty ratio

1 to 2 versus $>2$

$<1$ versus $>2$

Physical activity

inactive versus active \& meets guidelines

Active \& does not meet guidelines versus active \& meets guidelines

\begin{tabular}{|c|c|c|c|}
\hline 1.05 & 0.71 & 1.53 & 0.812 \\
\hline 2.68 & 1.51 & 4.75 & 0.001 \\
\hline 1.01 & 0.71 & 1.42 & 0.971 \\
\hline 2.50 & 1.86 & 3.37 & $<0.0001$ \\
\hline 3.85 & 2.79 & 5.31 & $<0.0001$ \\
\hline 0.82 & 0.70 & 0.97 & 0.021 \\
\hline 0.84 & 0.66 & 1.07 & 0.151 \\
\hline 1.38 & 1.01 & 1.90 & 0.044 \\
\hline 1.38 & 0.86 & 2.22 & 0.18 \\
\hline 1.18 & 0.96 & 1.44 & 0.106 \\
\hline 0.97 & 0.70 & 1.35 & 0.856 \\
\hline 0.60 & 0.34 & 1.04 & 0.067 \\
\hline 0.56 & 0.25 & 1.25 & 0.154 \\
\hline 0.67 & 0.55 & 0.82 & $<0.0001$ \\
\hline 1.10 & 0.88 & 1.36 & 0.388 \\
\hline 1.21 & 0.94 & 1.54 & 0.129 \\
\hline 1.40 & 1.08 & 1.82 & 0.012 \\
\hline 1.05 & 0.83 & 1.32 & 0.699 \\
\hline 1.09 & 0.75 & 1.57 & 0.641 \\
\hline 0.88 & 0.76 & 1.02 & 0.097 \\
\hline 0.82 & 0.64 & 1.06 & 0.123 \\
\hline 1.44 & 1.12 & 1.85 & 0.005 \\
\hline 2.27 & 1.85 & 2.78 & $<0.0001$ \\
\hline 2.14 & 1.61 & 2.85 & $<0.0001$ \\
\hline 1.06 & 0.86 & 1.30 & 0.604 \\
\hline 2.26 & 1.08 & 4.75 & 0.032 \\
\hline 1.41 & 0.82 & 2.43 & 0.213 \\
\hline 1.13 & 0.95 & 1.33 & 0.156 \\
\hline 1.12 & 0.94 & 1.33 & 0.187 \\
\hline 1.17 & 0.96 & 1.44 & 0.115 \\
\hline 0.87 & 0.68 & 1.13 & 0.297 \\
\hline 2.10 & 1.73 & 2.54 & $<0.0001$ \\
\hline 0.57 & 0.41 & 0.79 & 0.001 \\
\hline 0.97 & 0.78 & 1.22 & 0.795 \\
\hline 1.03 & 0.81 & 1.31 & 0.798 \\
\hline 0.97 & 0.76 & 1.23 & 0.777 \\
\hline 1.18 & 0.93 & 1.49 & 0.174 \\
\hline 1.06 & 0.89 & 1.26 & 0.533 \\
\hline
\end{tabular}


Table 3: Adjusted odds ratio and $95 \%$ confident intervals for significant associations with NAFLD based on multiple logistic regression for each racial/ethnic group.

\begin{tabular}{|c|c|c|c|c|c|c|c|c|c|c|c|c|}
\hline \multirow[b]{2}{*}{ Outcome: non alcoholic fatty liver } & \multicolumn{4}{|c|}{ White } & \multicolumn{4}{|c|}{ Black } & \multicolumn{4}{|c|}{ Mexican-American } \\
\hline & $\begin{array}{c}\text { Adjusted } \\
\text { Odds } \\
\text { Ratio }\end{array}$ & $\begin{array}{c}\text { Lower } 95 \% \\
\text { Confidence } \\
\text { level }\end{array}$ & $\begin{array}{c}\text { Upper } 95 \% \\
\text { confidence } \\
\text { level }\end{array}$ & $p$ - value & $\begin{array}{c}\text { Adjusted } \\
\text { Odds } \\
\text { Ratio }\end{array}$ & $\begin{array}{c}\text { Lower } 95 \% \\
\text { Confidence } \\
\text { level }\end{array}$ & $\begin{array}{c}\text { Upper } 95 \% \\
\text { confidence } \\
\text { level }\end{array}$ & $p$ - value & $\begin{array}{c}\text { Adjusted } \\
\text { Odds } \\
\text { Ratio }\end{array}$ & $\begin{array}{c}\text { Lower } 95 \% \\
\text { Confidence } \\
\text { level }\end{array}$ & $\begin{array}{c}\text { Upper } 95 \% \\
\text { confidence } \\
\text { level }\end{array}$ & $p$ - value \\
\hline \multicolumn{13}{|l|}{ Obesity phenotype } \\
\hline $\begin{array}{l}\text { Metabolically healthy overweight versus } \\
\text { metabolically healthy normal weight }\end{array}$ & 1.04 & 0.62 & 1.75 & 0.885 & 0.94 & 0.64 & 1.37 & 0.724 & 1.52 & 1.14 & 2.03 & 0.005 \\
\hline $\begin{array}{l}\text { Metabolically healthy obese versus metabolically } \\
\text { healthy normal weight }\end{array}$ & 3.31 & 1.48 & 7.41 & 0.004 & 1.25 & 0.72 & 2.15 & 0.422 & 1.59 & 0.88 & 2.88 & 0.123 \\
\hline $\begin{array}{l}\text { Metabolically unhealthy normal weight versus } \\
\text { metabolically healthy normal weight }\end{array}$ & 0.99 & 0.68 & 1.43 & 0.953 & 0.61 & 0.36 & 1.02 & 0.057 & 1.67 & 0.90 & 3.09 & 0.101 \\
\hline $\begin{array}{l}\text { Metabolically unhealthy overweight versus } \\
\text { metabolically healthy normal weight }\end{array}$ & 2.75 & 1.92 & 3.93 & 0.0001 & 0.87 & 0.57 & 1.33 & 0.511 & 2.06 & 1.37 & 3.09 & 0.001 \\
\hline $\begin{array}{l}\text { Metabolically unhealthy obese versus metabolically } \\
\text { healthy normal weight }\end{array}$ & 4.14 & 2.78 & 6.16 & 0.0001 & 1.86 & 1.32 & 2.63 & 0.001 & 3.85 & 2.33 & 6.38 & 0.0001 \\
\hline Female versus male & 0.89 & 0.74 & 1.07 & 0.212 & 0.86 & 0.60 & 1.23 & 0.408 & 0.69 & 0.53 & 0.89 & 0.006 \\
\hline Rural versus urban & 1.18 & 0.92 & 1.51 & 0.178 & 1.25 & 0.83 & 1.88 & 0.282 & 1.14 & 0.74 & 1.75 & 0.553 \\
\hline \multicolumn{13}{|l|}{ Language spoken at home } \\
\hline Spanish versus English & 0.78 & 0.10 & 6.22 & 0.81 & 0.75 & 0.14 & 3.93 & 0.731 & 1.05 & 0.77 & 1.44 & 0.755 \\
\hline Both English and Spanish versus English & 0.51 & 0.21 & 1.26 & 0.142 & 0.53 & 0.15 & 1.96 & 0.334 & 2.14 & 0.75 & 6.08 & 0.148 \\
\hline Other versus English & 1.00 & & & & 1.00 & & & & 1.06 & 0.66 & 1.70 & 0.815 \\
\hline \multicolumn{13}{|l|}{ Smoking status } \\
\hline Current versus never & 0.68 & 0.53 & 0.86 & 0.002 & 0.65 & 0.49 & 0.87 & 0.005 & 0.66 & 0.49 & 0.90 & 0.01 \\
\hline Former versus never & 1.11 & 0.86 & 1.44 & 0.416 & 0.95 & 0.69 & 1.31 & 0.756 & 1.12 & 0.89 & 1.40 & 0.324 \\
\hline \multicolumn{13}{|l|}{ Age group (years) } \\
\hline $35-49$ versus $20-34$ years & 1.18 & 0.86 & 1.61 & 0.302 & 1.07 & 0.79 & 1.45 & 0.667 & 1.58 & 1.18 & 2.13 & 0.003 \\
\hline $50+$ versus $20-34$ years & 1.36 & 0.98 & 1.91 & 0.069 & 1.26 & 0.87 & 1.81 & 0.212 & 1.42 & 1.02 & 1.97 & 0.038 \\
\hline \multicolumn{13}{|l|}{ Healthy eating index group } \\
\hline needs improvement versus good & 1.06 & 0.81 & 1.39 & 0.672 & 1.61 & 0.93 & 2.80 & 0.088 & 1.10 & 0.83 & 1.45 & 0.498 \\
\hline poor diet versus good & 1.14 & 0.72 & 1.80 & 0.573 & 1.78 & 0.93 & 3.39 & 0.081 & 0.94 & 0.70 & 1.27 & 0.677 \\
\hline \multicolumn{13}{|l|}{ Cholesterol groups } \\
\hline elevated $(200-239 \mathrm{mg})$ versus normal & 0.83 & 0.68 & 1.00 & 0.049 & 1.22 & 0.93 & 1.59 & 0.149 & 0.95 & 0.73 & 1.24 & 0.695 \\
\hline high (>=240 mg/dL) versus normal & 0.77 & 0.58 & 1.02 & 0.071 & 1.36 & 0.96 & 1.92 & 0.08 & 0.99 & 0.78 & 1.25 & 0.912 \\
\hline \multicolumn{13}{|l|}{ Triglyceride groups } \\
\hline borderline (150-199) versus normal & 1.56 & 1.14 & 2.14 & 0.007 & 1.33 & 0.89 & 1.98 & 0.156 & 1.16 & 0.75 & 1.81 & 0.495 \\
\hline high (>=200 mg/dL) versus normal & 2.28 & 1.79 & 2.91 & $<0.0001$ & 1.86 & 1.29 & 2.67 & 0.001 & 1.39 & 1.03 & 1.88 & 0.034 \\
\hline \multicolumn{13}{|l|}{ Glucose groups } \\
\hline diabetes (>125 mg/dL) versus normal & 2.19 & 1.52 & 3.16 & $<0.0001$ & 2.02 & 1.31 & 3.09 & 0.002 & 2.20 & 1.51 & 3.20 & $<0.0001$ \\
\hline prediabetes (100-125) versus normal & 0.95 & 0.73 & 1.25 & 0.729 & 1.16 & 0.86 & 1.55 & 0.326 & 1.29 & 0.91 & 1.82 & 0.155 \\
\hline \multicolumn{13}{|l|}{ ALT groups } \\
\hline elevated (>56 U/L) versus normal & 2.82 & 0.92 & 8.66 & 0.069 & 0.82 & 0.26 & 2.63 & 0.733 & 2.06 & 1.28 & 3.33 & 0.004 \\
\hline \multicolumn{13}{|l|}{ AST groups } \\
\hline elevated (>40 U/L) versus normal & 1.50 & 0.70 & 3.25 & 0.292 & 1.56 & 0.87 & 2.80 & 0.129 & 1.51 & 0.89 & 2.58 & 0.123 \\
\hline \multicolumn{13}{|l|}{ Education level } \\
\hline high school versus more than high school & 1.10 & 0.90 & 1.36 & 0.339 & 1.04 & 0.81 & 1.34 & 0.736 & 0.98 & 0.66 & 1.47 & 0.936 \\
\hline less than high school versus more than high school & 1.10 & 0.86 & 1.41 & 0.45 & 1.18 & 0.84 & 1.65 & 0.335 & 0.99 & 0.70 & 1.39 & 0.952 \\
\hline \multicolumn{13}{|l|}{ CRP groups } \\
\hline mild inflammation versus normal & 1.15 & 0.87 & 1.51 & 0.321 & 1.24 & 0.96 & 1.60 & 0.091 & 1.22 & 0.97 & 1.54 & 0.083 \\
\hline significant inflammation versus normal & 0.85 & 0.62 & 1.15 & 0.283 & 1.10 & 0.79 & 1.53 & 0.569 & 0.75 & 0.54 & 1.04 & 0.084 \\
\hline C-peptide groups & & & & & & & & & & & & \\
\hline high (>1.03 nmol/L) versus normal & 2.20 & 1.72 & 2.81 & $<0.0001$ & 1.87 & 1.44 & 2.44 & $<0.0001$ & 1.93 & 1.40 & 2.65 & $<0.0001$ \\
\hline low $(<.26 \mathrm{nmol} / \mathrm{L})$ versus normal & 0.52 & 0.35 & 0.76 & 0.001 & 0.70 & 0.46 & 1.06 & 0.093 & 0.41 & 0.22 & 0.79 & 0.009 \\
\hline Hypertension & & & & & & & & & & & & \\
\hline yes versus no & 1.06 & 0.83 & 1.36 & 0.625 & 1.02 & 0.73 & 1.43 & 0.901 & 1.00 & 0.71 & 1.40 & 0.99 \\
\hline Poverty ratio & & & & & & & & & & & & \\
\hline 1 to 2 versus $>2$ & 0.96 & 0.69 & 1.34 & 0.815 & 1.10 & 0.75 & 1.62 & 0.616 & 1.21 & 0.89 & 1.64 & 0.215 \\
\hline$<1$ versus $>2$ & 0.94 & 0.64 & 1.37 & 0.733 & 1.09 & 0.78 & 1.53 & 0.61 & 1.14 & 0.81 & 1.61 & 0.455 \\
\hline Physical activity & & & & & & & & & & & & \\
\hline inactive versus active $\&$ meets guidelines & 1.24 & 0.90 & 1.71 & 0.187 & 1.04 & 0.76 & 1.42 & 0.813 & 1.11 & 0.82 & 1.51 & 0.496 \\
\hline $\begin{array}{l}\text { Active \& does not meet guidelines versus active \& } \\
\text { meets guidelines }\end{array}$ & 1.04 & 0.84 & 1.29 & 0.694 & 1.18 & 0.85 & 1.62 & 0.31 & 1.00 & 0.84 & 1.18 & 0.964 \\
\hline
\end{tabular}


group, while only Hispanics had increased odds of NAFLD in the metabolically healthy overweight group. These results highlight the importance of considering racial/ethnic group in how the obesity phenotype affects risk for NAFLD.

Previous studies have not evaluated race/ethnicity as a factor in the relationship between obesity phenotype and hepatic steatosis. However, other studies have tended to find that NAFLD prevalence is highest in Hispanics and lowest in Blacks [18,20]. Various factors are thought to account for racial/ethnic differences in risk for and prognosis of NAFLD, including differences in socio-economic status and access to care [20]. Genetic factors, in particular, are thought to have a major influence on risk and severity. For example, polymorphisms of PNPLA3, TM6SF2, and MBOAT are associated with risk for NAFLD and are distributed unequally among different racial/ethnic groups, providing potential mechanisms for the observed racial disparities [20,21]. Additionally, one study found that a certain polymorphism of PNPLA3 seems to be more involved in progression of NAFLD in non-obese individuals than obese individuals [33], while another study found that carriers of the polymorphism were less likely to have metabolic syndrome [34]. In another study, while metabolic syndrome was associated with an increased risk of NAFLD-related mortality, polymorphisms of PNPLA3, TM6SF2, and MBOAT were not [35]. The interactions between these genetic factors may provide a potential mechanism for interaction between the effects of race/ethnicity and obesity phenotype on risk for NAFLD. Further research is needed to illuminate the mechanism underlying this interaction.

\section{Limitations}

Our study had some limitations. First, hepatic steatosis data from NHANES III (1988-1994) were based on ultrasound files. The sensitivity, specificity, and accuracy of ultrasound has been shown to be $85 \%, 94 \%$, and $93 \%$ when compared to liver biopsy [36]. In regards to diagnoses made through imaging, the literature has established that imaging tests such as ultrasounds and CT scans are unable to differentiate between hepatic fibrosis from simple hepatic steatosis as seen in patients with NAFLD [2]. Ultrasonography has also been criticized for low sensitivity to mild steatosis and poor discrimination between moderate and severe steatosis, but since our steatosis definition did not include mild levels and combined moderate and severe, these concerns would not affect our results. Newer methods such as transient liver elastography can differentiate between hepatic steatosis and hepatic fibrosis with better accuracy than ultrasound. But NHANES III remains the only nationally representative sample that allows for identification of NAFLD. A related limitation is that because of the way we categorized steatosis groups, we did not assess degree of severity of hepatic steatosis or fibrosis.

A second limitation was how the obesity phenotype was defined in this study. There is no universal definition for this phenotype. As a result, other definitions might have resulted in different outcomes. While the metabolic syndrome has a more consistent definition, there may still be some differences in the various cutoffs used. It is well known that a variety of factors exist amongst different ethnic groups which can determine metabolic health and can involve other markers not addressed in this study.

A third limitation is that the survey design was crosssectional study so we cannot make causal inference and determine the mechanism of the association. We can only determine an association between NAFLD and the obesity phenotype. Furthermore, some of the variables were measured by self-report where there is a possibility of recall bias.

\section{Conclusion}

This study shows that an independent relationship exists between the obesity phenotype and NAFLD. The metabolically healthy obese had a high chance of NAFLD. The odds of NAFLD were higher in those who are part of the metabolically unhealthy overweight and obese groups compared to those in the metabolically healthy normal group. Furthermore, there were differences by race/ethnicity. The prevalence of NAFLD was highest in Mexican-Americans when compared to Whites. Also, only in Mexican-Americans, the metabolic healthy overweight groups had a higher chance of developing NAFLD. These findings support the hypothesis that the prevalence of NAFLD is higher among the metabolically healthy overweight and obese phenotypes in the US population.

\section{Recommendations}

Therefore, healthcare providers should pay more attention to care for those who are part of the metabolically healthy overweight or obese group, especially among the MexicanAmerican population. We recommend further research to explore the possible mechanism of the relation between NAFLD and obesity phenotype.

\section{Acknowledgement}

This research was funded by NIH-NIMHD grants U54MD007598 and S21MD000103, and NIH/NCATS grant UL1TR000124.

\section{References}

1. Dietrich $P$, Hellerbrand $C$. Non-alcoholic fatty liver disease, obesity and the metabolic syndrome. Best Pract Res Clin Gastroenterol. 2014; 28 : 637-653.

PubMed: https://pubmed.ncbi.nlm.nih.gov/25194181/

2. Chalasani N, Younossi Z, Lavine JE, Charlton M, Cusi K, et al. The diagnosis and management of nonalcoholic fatty liver disease: Practice guidance from the American Association for the Study of Liver Diseases. Hepatology. 2018; 67: 328-357.

PubMed: https://pubmed.ncbi.nlm.nih.gov/28714183/

3. Anstee QM, Targher G, Day CP. Progression of NAFLD to diabetes mellitus, cardiovascular disease or cirrhosis. Nat Rev Gastroenterol 
Hepatol. 2013; 10: 330-344.

PubMed: https://pubmed.ncbi.nlm.nih.gov/23507799/

4. Kim HJ, Kim HJ, Lee KE, Kim DJ, Kim SK, et al. Metabolic significance of nonalcoholic fatty liver disease in nonobese, nondiabetic adults. Arch Intern Med. 2004; 164: 2169-2175.

PubMed: https://pubmed.ncbi.nlm.nih.gov/15505132/

5. Marchesini G, Marzocchi R. Metabolic syndrome and NASH. Clin Liver Dis. 2007; 11: 105-117.

PubMed: https://pubmed.ncbi.nlm.nih.gov/17544974/

6. Lonardo A, Ballestri S, Marchesini G, Angulo P, Loria P. Nonalcoholic fatty liver disease: a precursor of the metabolic syndrome. Digestive and Liver Disease. 2015; 47: 181-190.

PubMed: https://pubmed.ncbi.nlm.nih.gov/25739820/

7. Marchesini G, Bugianesi E, Forlani G, Cerrelli F, Lenzi M, et al. Nonalcoholic fatty liver, steatohepatitis, and the metabolic syndrome. Hepatology. 2003; 37: 917-923.

PubMed: https://pubmed.ncbi.nlm.nih.gov/12668987/

8. Cotrim HP, Parise ER, Oliveira CP, Leite N, Martinelli A, et al. Nonalcoholic fatty liver disease in Brazil. Clinical and histological profile. Ann Hepatol. 2011; 10: 33-37.

PubMed: https://pubmed.ncbi.nlm.nih.gov/21301007/

9. Goossens GH. The Metabolic Phenotype in Obesity: Fat Mass, Body Fat Distribution, and Adipose Tissue Function. Obes Facts. 2017; 10: 207-215.

PubMed: https://pubmed.ncbi.nlm.nih.gov/28564650/

10. Park J, Kim SH, Cho GY, Baik I, Kim NH, et al. Obesity phenotype and cardiovascular changes. J Hypertens. 2011; 29: 1765-1772.

PubMed: https://pubmed.ncbi.nlm.nih.gov/21826021/

11. Meigs JB, Wilson PW, Fox CS, Vasan RS, Nathan DM, et al. Body mass index, metabolic syndrome, and risk of type 2 diabetes or cardiovascular disease. J Clin Endocrinol Metabol. 2006; 91: 2906-2912. PubMed: https://pubmed.ncbi.nlm.nih.gov/16735483/

12. Kabootari M, Akbarpour S, Azizi F, Hadaegh F. Sex specific impact of different obesity phenotypes on the risk of incident hypertension: Tehran lipid and glucose study. Nutrition Metabol. 2019; 16: 16.

13. Chang Y, Jung HS, Yun KE, Cho J, Ahn J, et al. Metabolically healthy obesity is associated with an increased risk of diabetes independently of nonalcoholic fatty liver disease. Obesity. 2016; 24: 1996-2003. PubMed: https://pubmed.ncbi.nlm.nih.gov/27474900/

14. Shaharyar S, Roberson LL, Jamal O, Younus A, Blaha MJ, et al. Obesity and metabolic phenotypes (metabolically healthy and unhealthy variants) are significantly associated with prevalence of elevated C-reactive protein and hepatic steatosis in a large healthy Brazilian population. J Obes. 2015; 2015. 178526.

PubMed: https://pubmed.ncbi.nlm.nih.gov/25838943/

15. Sinn DH, Gwak GY, Park HN, Kim JE, Min YW, etal. Ultrasonographically detected non-alcoholic fatty liver disease is an independent predictor for identifying patients with insulin resistance in non-obese, non-diabetic middle-aged Asian adults. Am J Gastroenterol. 2012; 107: 561-567. PubMed: https://pubmed.ncbi.nlm.nih.gov/22108448/

16. Stefan N, Schick F, Haering HU. Causes, characteristics, and consequences of metabolically unhealthy normal weight in humans. Cell Metabol. 2017; 26: 292-300.

PubMed: https://pubmed.ncbi.nlm.nih.gov/28768170/

17. Younossi Z, Anstee QM, Marietti M, Hardy T, Henry L, et al. Global burden of NAFLD and NASH: trends, predictions, risk factors and prevention. Nat Rev Gastroenterol Hepatol. 2018; 15: 11-20.

PubMed: https://pubmed.ncbi.nlm.nih.gov/28930295/

18. Younossi ZM, Stepanova M, Negro F, Hallaji S, Younossi $Y$, et al Nonalcoholic fatty liver disease in lean individuals in the United States. Medicine (Baltimore). 2012; 91: 319-327.

PubMed: https://pubmed.ncbi.nlm.nih.gov/23117851/
19. Browning JD, Kumar KS, Saboorian MH, Thiele DL. Ethnic differences in the prevalence of cryptogenic cirrhosis. Am J Gastroenterol. 2004; 99: 292-298.

PubMed: https://pubmed.ncbi.nlm.nih.gov/15046220/

20. Rich NE, Oji S, Mufti AR, Browning JD, Parikh ND, et al. Racial and Ethnic Disparities in Nonalcoholic Fatty Liver Disease Prevalence, Severity, and Outcomes in the United States: A Systematic Review and Meta-analysis. Clin Gastroenterol Hepatol. 2018; 16: 198-210. PubMed: https://pubmed.ncbi.nlm.nih.gov/28970148/

21. Romeo S, Kozlitina J, Xing C, Pertsemlidis A, Cox D, et al. Genetic variation in PNPLA3 confers susceptibility to nonalcoholic fatty liver disease. Nat Genet. 2008; 40: 1461-1465.

PubMed: https://pubmed.ncbi.nlm.nih.gov/18820647/

22. Petersen R, Pan L, Blanck HM. Peer Reviewed: Racial and Ethnic Disparities in Adult Obesity in the United States: CDC's Tracking to Inform State and Local Action. Prevent Chronic Dis. 2019; 16: E46. PubMed: https://pubmed.ncbi.nlm.nih.gov/30974071/

23. Ford ES, Giles WH, Dietz WH. Prevalence of the Metabolic Syndrome Among US AdultsFindings From the Third National Health and Nutrition Examination Survey. JAMA. 2002; 287: 356-359. PubMed: https://pubmed.ncbi.nlm.nih.gov/11790215/

24. Heiss G, Snyder ML, Teng Y, Schneiderman N, Llabre MM, et al. Prevalence of Metabolic Syndrome Among Hispanics/Latinos of Diverse Background: The Hispanic Community Health Study/Study of Latinos. Diabetes Care. 2014; 37: 2391-2399.

PubMed: https://pubmed.ncbi.nlm.nih.gov/25061141/

25. Ervin RB. Prevalence of metabolic syndrome among adults 20 years of age and over, by sex, age, race and ethnicity, and body mass index; United States. 2003-2006. Natl Health Stat Report. 2009; 5: 1-7. PubMed: https://pubmed.ncbi.nlm.nih.gov/19634296/

26. World Medical Association Declaration of Helsinki: ethical principles for medical research involving human subjects. JAMA. 2013; 310: 2191-2194. PubMed: https://pubmed.ncbi.nlm.nih.gov/24141714/

27. Plan and operation of the Third National Health and Nutrition Examination Survey, 1988-94. Series 1: programs and collection procedures. Vital Health Stat 1. 1994: 1-407. PubMed: https://pubmed.ncbi.nlm.nih.gov/7975354/

28. Ruhl CE, Everhart JE. Fatty liver indices in the multiethnic united states national health and nutrition examination survey. Alimentary Pharmacol Therapeutics. 2015; 41: 65-76.

PubMed: https://pubmed.ncbi.nlm.nih.gov/25376360/

29. Golabi P, Otgonsuren M, de Avila L, Sayiner M, Rafiq N, et al. Components of metabolic syndrome increase the risk of mortality in nonalcoholic fatty liver disease (NAFLD). Medicine. 2018; 97: e0214. PubMed: https://pubmed.ncbi.nlm.nih.gov/29595666/

30. Ferreira VSG, Pernambuco RB, Lopes EP, Morais CN, Rodrigues MC, et al. Frequency and risk factors associated with non-alcoholic fatty liver disease in patients with type 2 diabetes mellitus. Arq Bras Endocrinol Metabol. 2010; 54: 362-368.

https://pubmed.ncbi.nlm.nih.gov/20625647/

31. Sung KC, Cha SC, Sung JW, So MS, Byrne CD. Metabolically healthy obese subjects are at risk of fatty liver but not of pre-clinical atherosclerosis. Nutrit Metabol Cardiovasc Dis. 2014; 24: 256-262. PubMed: https://pubmed.ncbi.nlm.nih.gov/24361070/

32. Huh JH, Kim KJ, Kim SU, Han SH, Han KH, et al. Obesity is more closely related with hepatic steatosis and fibrosis measured by transient elastography than metabolic health status. Metabolism. 2017; 66: 23-31. PubMed: https://pubmed.ncbi.nlm.nih.gov/27923446/

33. Lu FB, Hu ED, Xu LM, Chen L, Wu JL, Li H, et al. The relationship between obesity and the severity of non-alcoholic fatty liver disease: systematic review and meta-analysis. Expert Review of Gastroenterol Hepatol. 2018; 12: 491-502.

PubMed: https://pubmed.ncbi.nlm.nih.gov/29609501/ 
34. Lonardo A, Ballestri S, Targher G. "Not all forms of NAFLD were created equal". Do metabolic syndrome-related NAFLD and PNPLA3related NAFLD exert a variable impact on the risk of early carotid atherosclerosis? Atherosclerosis. 2017; 257: 253-255.

PubMed: https://pubmed.ncbi.nlm.nih.gov/28126321/

35. Käräjämäki AJ, Hukkanen J, Kauma $H$, Kesäniemi YA, Ukkola $O$. Metabolic syndrome but not genetic polymorphisms known to induce NAFLD predicts increased total mortality in subjects with NAFLD
(OPERA study). Scandinavian J Clin Laboratory Investigation. 2020; 80: 106-113.

PubMed: https://pubmed.ncbi.nIm.nih.gov/31851849/

36. Hernaez R, Lazo M, Bonekamp S, Kamel I, Brancati FL, et al. Diagnostic accuracy and reliability of ultrasonography for the detection of fatty liver: a meta-analysis. Hepatology. 2011; 54: 1082-1090. PubMed: https://pubmed.ncbi.nlm.nih.gov/21618575/ 